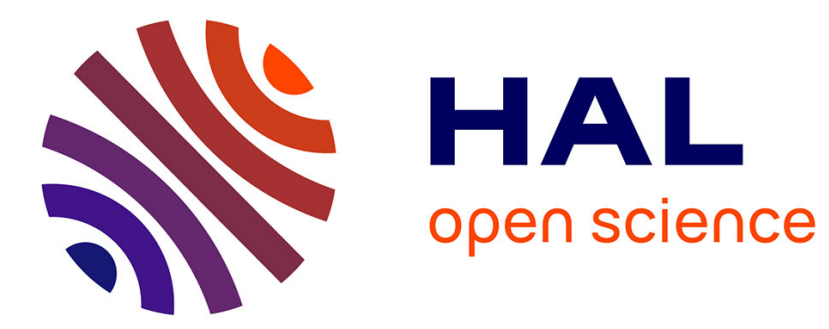

\title{
Camphor odor recognition within unbalanced multi-sets
}

Nouha Chaoued, Amel Borgi, Anne Laurent

\section{To cite this version:}

Nouha Chaoued, Amel Borgi, Anne Laurent. Camphor odor recognition within unbalanced multisets. FUZZ-IEEE 2017 - International Conference on Fuzzy Systems, Jul 2017, Naples, Italy. pp.1-6, 10.1109/FUZZ-IEEE.2017.8015540 . hal-01847702

\section{HAL Id: hal-01847702 \\ https://hal.science/hal-01847702}

Submitted on 8 Oct 2019

HAL is a multi-disciplinary open access archive for the deposit and dissemination of scientific research documents, whether they are published or not. The documents may come from teaching and research institutions in France or abroad, or from public or private research centers.
L'archive ouverte pluridisciplinaire HAL, est destinée au dépôt et à la diffusion de documents scientifiques de niveau recherche, publiés ou non, émanant des établissements d'enseignement et de recherche français ou étrangers, des laboratoires publics ou privés. 


\title{
Camphor Odor Recognition within Unbalanced Multi-Sets
}

\author{
Nouha Chaoued ${ }^{* \ddagger}$, Amel Borgi ${ }^{\dagger}$, Anne Laurent ${ }^{\ddagger}$ \\ *Université de Tunis El Manar, Faculté des Sciences de Tunis, LIPAH - LR 11 ES 14, 1002, Tunis, Tunisie \\ †Université de Tunis El Manar, Institut Supérieur d'Informatique, Faculté des Sciences de Tunis, \\ LIPAH LR 11 ES 14, 1002, Tunis, Tunisie \\ Email: Amel.Borgi@insat.rnu.tn \\ $\ddagger$ Université de Montpellier, LIRMM - CNRS UMR 5506, F-34392 Montpellier, France \\ Email: firstname.lastname@lirmm.fr
}

\begin{abstract}
In fuzzy logic context, some works deal with the camphor odor perception. In this paper, we present a novel rule-based decision system for the camphor odor recognition within unbalanced multiset. Our first contribution consists in an adaptation of fuzzy knowledge representation and inference rules to the multi-valued logic context. The second contribution concerns the improvement of the knowledge base by changing facts representation and adding new rules. This proposition provides satisfactory results in term of precision, recall, F-measure and accuracy.
\end{abstract}

\section{INTRODUCTION}

Alexander Graham Bell said a century ago, "until you can measure their likenesses and differences you can have no science of odor" [1]. Even so, scientists have tended to predict or identify odors based on their molecular structure.

Several factors influence odor perception as the external environment and the individual. The recognition of an odor by humans results from a stimulus. It is not objective as it is affected by the cognitive interpretation.

Basically, odor is quantified by three parameters: quality or identity, intensity or strength, hedonic character [2]. Many methods have been used to quantify odor. But, these methods are affected by different problems as the detection cost, the long time took for analysis and detection, and exposing human to danger.

Artificial intelligence algorithms and techniques such as fuzzy logic, neural networks, and genetic algorithms had improved the process of decision-making in odor perception systems [3].

The authors in [4] indicate that the performance of fuzzy logic approaches depends on the used membership function. The universe of discourse in the fuzzy context may be discrete or qualitative but more often it is numerical. Furthermore, the determination of fuzzy membership functions is not always trivial.

In this paper, we focus on multi-valued logic. It allows to symbolically represent imprecise knowledge using ordered linguistic symbols from natural language [5]. Multi-valued logic allows the knowledge representation regardless of their reference domain type.

\section{PRELiminaries}

Multi-valued logic is based on De Glas's theory [5]. Linguistic variables are used to represent an opinion independent of a measuring system. In this approach, every linguistic variable is represented by an ordered and finite list of $M$ linguistic symbols. They constitute a multi-set denoted by [5] [6]:

$$
L_{M}=\left\{\tau_{0}, \tau_{1}, \ldots, \tau_{M-1}\right\} ; M \in \mathbb{N}^{*} \backslash\{1\}
$$

$\tau_{i}$ is the membership degree to the multi-set $L_{M}$ with $i \in[0, M-1]$. The membership relation in multi-valued logic is partial:

$$
x \in \in_{\alpha} A \Leftrightarrow x \text { belongs to } A \text { at a degree } \alpha
$$

It should be noted that symbolic degrees are connected only by the total order relation $\leq$ defined by $[7]$ :

$$
\tau_{\alpha} \leq \tau_{\beta} \Leftrightarrow \alpha \leq \beta ; \forall \alpha \text { and } \beta \in[0, M-1]
$$

A qualifier $\vartheta_{\alpha}$ is associated with each degree to express the imprecision of a predicate. A predicate is a feature of language used to attribute a property to a thing.

$$
\begin{aligned}
x \text { is } \vartheta_{\alpha} A & \Longleftrightarrow\left(x \text { is } \vartheta_{\alpha} A\right) \text { is true } \\
& \Longleftrightarrow(x \text { is } A) \text { is } \tau_{\alpha} \text { true }
\end{aligned}
$$

For example saying that the odor is little pleasant means that the odor satisfies the predicate pleasant with the degree $\tau_{1}$ (Figure 1).

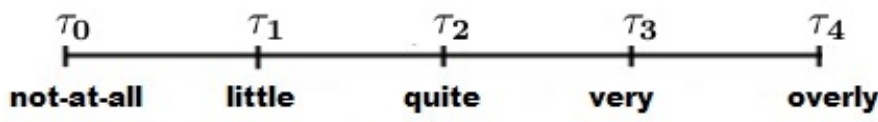

Figure 1. Representation of a scale of 5 truth-degrees

Linguistic term sets are usually assumed to be uniformly distributed as the distance between every pair of successive terms is the same, i.e. $\frac{1}{M-1}$ with $M$ the number of terms in the set. Nevertheless, in some cases, a particular subdomain may be more informative than the remaining 


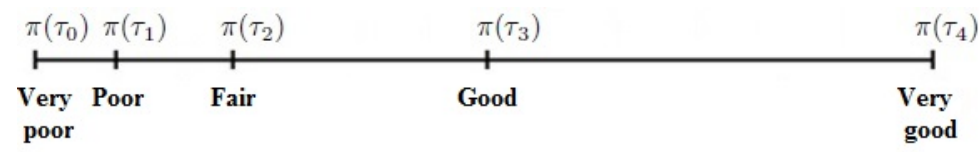

(a) Terms position

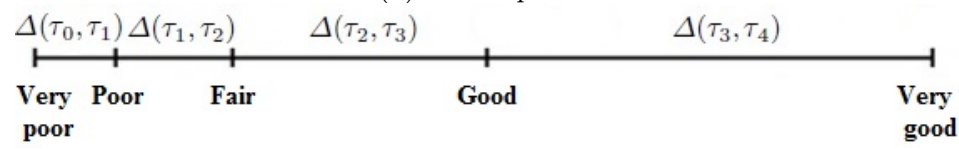

(b) Distances between terms

Figure 2. Terms Definitions

reference domain. Thus, the density of terms in this subdomain is greater then in the others.

These linguistic term sets are said to be unbalanced, knowsn as unbalanced sets. Some authors [8]-[13] proposed examples from real life using these sets. Truck [14] indicates that such sets "are commonly used to describe erratic phenomenons and irregular progression in the patterns such as weather events or behavior under the influence of alcohol". Xia and $\mathrm{Xu}$ [15] give the example of diminishing marginal utility in economics. They indicate that the investment of a same amount is different if the company has a bad or a good performance. In this case, we need more precise bad information than good one. Hence, the gaps between the grades expressing bad information are smaller than the ones between the grades expressing good information.

In our work, we deal with unbalanced terms set in the context of multi-valued logic. This multi-set is defined as: $L_{M}=\left\{\tau_{0}, \tau_{1}, \ldots, \tau_{(M-1)}\right\} ; M \in \mathbb{N}^{*} \backslash\{1\}$; with $M$ its cardinality.

Each term $\tau_{i}$ represents a possible value for a linguistic variable. It is defined by its position $\pi\left(\tau_{i}\right)$ (Figure 2-a):

$$
\pi: L_{M} \rightarrow[0,1]
$$

This function represents the distribution of the terms $\tau_{i}$ using real values between 0 and 1 .

These terms are not necessarily symmetrically distributed or equidistant from each others, i.e. $\Delta\left(\tau_{i}, \tau_{i+1}\right)$ are not equal for all $i \in[0, M-1]$ (Figure 2-b):

$$
\begin{gathered}
\Delta: L_{M} \times L_{M} \rightarrow[0,1] \\
\Delta\left(\tau_{i}, \tau_{j}\right)=\pi\left(\tau_{j}\right)-\pi\left(\tau_{i}\right)
\end{gathered}
$$

In this paper, we implement an inference engine for camphor odor perception. Factual knowledge and used rules are expressed within unbalanced multi-sets.

\section{INFERENCE ENGINE}

An expert system consists of a knowledge base and an inference engine. It uses knowledge about its application domain, i.e. factual knowledge, and a reasoning process based on heuristic knowledge to solve problems that usually require human expertise.

Application areas include classification, diagnosis, monitoring, process control, design, scheduling and planning, and generation of options. In this work, we are interested in classification systems which allow to identify an object based on its characteristics.

An inference engine allows reasoning by applying logical rules to the knowledge base to deduct new knowledge. This process is iterated when a new fact in the knowledge base could trigger additional rules in the inference engine.

Approximate reasoning allows inference with approximate values and incomplete or ambiguous data. It lets us dealing with uncertainty in some expert systems and gives us more flexible handling of problems. It was defined by Zadeh in fuzzy logic context [16] as a set of methods that allow reasoning with imprecise inputs to obtain meaningful outputs. It is based on the Generalized Modus Ponens (GMP).

Reasoning uses production rules that consist of an IF part, i.e. a condition or premise, and a THEN part, i.e. an action or conclusion.

In the multi-valued logic context, the authors in [17][19] have proposed different models with if-then rules. In the literature, rules are classified as: free or strong, simple or complex.

In this work, we use an adaptation of the GMP model proposed in [19], [20] to infer within unbalanced multi-sets. This model deals with strong complex rules. The GMP model is as follow:

$$
\begin{array}{r}
\text { If }\left(X_{1} \text { is } \vartheta_{\alpha_{1}} A_{1}\right) \text { and } \ldots \text { and }\left(X_{n} \text { is } \vartheta_{\alpha_{n}} A_{n}\right) \\
\text { Then }\left(Y \text { is } \vartheta_{\beta} B\right) \\
\left(X_{1} \text { is } \vartheta_{\gamma_{1}} A_{1}\right) \text { and } \ldots \text { and }\left(X_{n} \text { is } \vartheta_{\gamma_{n}} A_{n}\right)
\end{array}
$$

With:

$$
\left(Y \text { is } \vartheta_{\lambda} B\right)
$$

- $A_{1}, A_{2}, \ldots, A_{n}$ are the rule predicates representing the premise and the observation, i.e. fact;

- $B$ is the predicate representing the rule conclusion;

- $A_{i}$ and $B$ are represented by the unbalanced multisets $L_{M_{A_{i}}}(\mathrm{i} \in\{1, \ldots \mathrm{n}\})$ and $L_{M_{B}}$ respectively;

- $n$ is the number of elements in the rule premise.

The inference engine aims to deduct the inferred conclusion $\vartheta_{\lambda} B$. Our inference engine is based on forward chaining mode. The process starts with the known facts and deduce new facts. 


\section{ODORS CLASSIFIER}

The qualification of odors and the measurement of theirs intensities are based on human perception and are therefore subjective. The lack of numerical information due to the unavailability of measures for odors is the first problem when studying odors. Every odor has threedimensional different characteristics which are difficult to separate [2]:

- Quality or identity: as floral, fruity, musk or camphor;

- Intensity or strength: strong, medium, weak;

- Hedonic character: pleasant, unpleasant.

The semantic descriptors of odors are numerous, not precise, often referring to other odors, themselves described by a set of qualitative descriptors.

For this reason, we propose a tool for odor perception within multi-valued logic. We have implemented an inference engine based on the generic Knowledge Based System RAMOLI proposed in [21]. RAMOLI provides a reasoning process within uniform multi-sets. Our system aims to classify molecules according to the intensity of camphor odor within unbalanced multi-sets.

The olfactory properties of molecules have been described by Schnabel et al. [22]:

- $\mathbf{R}$ : the molecular length;

- $\mathbf{r}$ : the molecular width;

- $\mathbf{R} / \mathbf{r}$ : the measure of sphericity of the molecule;

- $\mathbf{S}$ : the measure of the surface of the molecule;

- d: the density of the molecule (weight/volume).

The Schnabel score classifies molecules into three classes:

- non camphorated when the score is 0 ;

- slightly camphorated for ones with a score between 1 and 2 ;

- very camphorated when the score is between 3 and 5 .

The set of studied compounds includes 99 aliphatic alcohols: 29 primary, 52 secondary and 18 tertiary, with one or two carbon atoms.

To achieve the classification, the system uses facts within a multi-valued representation. To do so, we discretize the attribute values describing the molecules. We replace each numerical value with an equivalent linguistic term. Every molecule corresponds to a fact in the knowledge base.

Kissi, Ramdani et al. [23] [24] proposed a representation of the membership functions for the linguistic variables spherical (Figure 3), surface (Figure 4) and camphorated (Figure 5) in the fuzzy logic context. spherical

not spherical

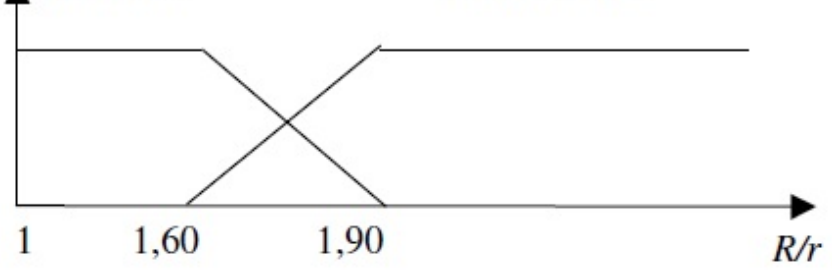

Figure 3. Membership functions for linguistic variable Spherical

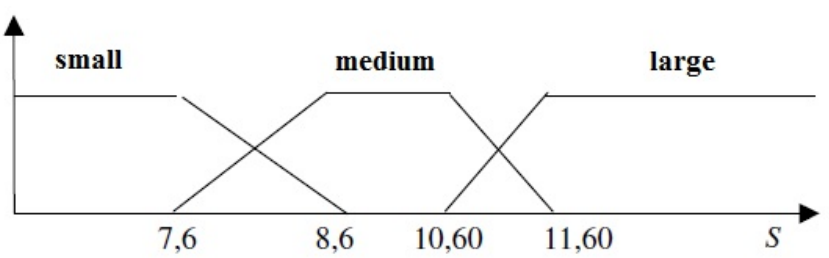

Figure 4. Membership functions for linguistic variable Surface

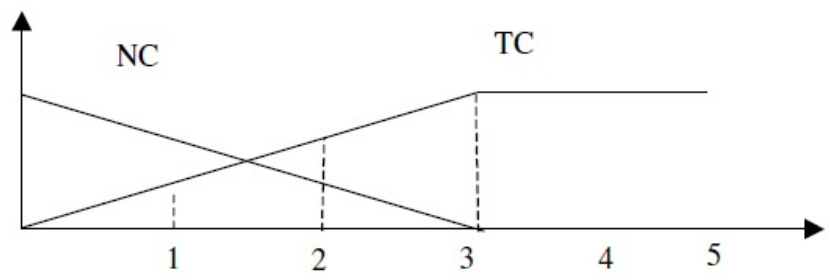

Figure 5. Membership functions for linguistic variable Camphorated

We notice that these fuzzy sets are unbalanced.

In a multi-valued logic context, we propose to represent these variables within the following unbalanced multi-sets:

- Spherical: $L_{3}^{s}=\{$ surely, almost, not $\}$ (Figure 6), with $\pi($ almost $)=0.3$;

- Surface: $L_{4}=\{$ small, medium, large, very large $\}$ (Figure $7)$, with $\pi($ medium $)=0.62$ and $\pi($ large $)=0.85$;

- Camphorated: $L_{3}^{c}=\{$ not, slightly, very $\}$ (Figure 8), with $\pi($ slightly $)=0.6$.

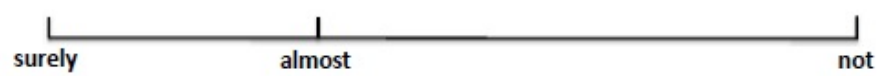

Figure 6. Representation of Spherical Multi-set

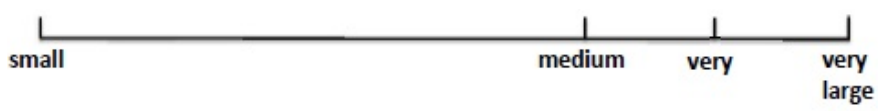

Figure 7. Representation of Surface Multi-set

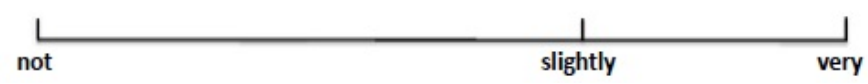

Figure 8. Representation of Camphorated Multi-set 
The variable density is added by the authors in [23] [24] to improve the accuracy of their system. Its corresponding multi-set is: $L_{3}^{d}=$ \{low, moderately, high $\}$ (Figure 9), with $\pi$ (moderately $)=0.802$.

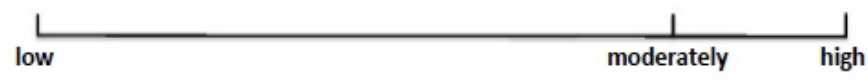

Figure 9. Representation of Dense Multi-set

During the classification process, the facts are compared to the premise of existing rules. The rule triggered by the inference engine is that whose premise is the most similar to the fact.

\section{Experimental Tests}

To evaluate our inference engine, we proceed to three tests for camphor odor recognition. In the two first ones, we adapt to the multi-valued logic context the molecules representation and the fuzzy rules set proposed in [23] [24]. In the third test, we propose new molecules representation and new rules based on the set of studied compounds.

All these tests use unbalanced multi-sets.

\section{A. First Test}

The authors in [23] [24] describe each molecule with the attributes: surface (small, medium, large) and spherical $(y e s, n o)$. The camphor odor can be not or very.

The expert classification rules proposed in [23] [24] are:

- If molecule is spherical and medium then its odor is very camphorated

- If molecule is spherical and small then its odor is not camphorated

- If molecule is spherical and large then its odor is not camphorated

- If molecule is not spherical then its odor is not camphorated

In this first test, in multi-valued logic context, we use the multi-sets $L_{3}^{s}$ (Figure 6) and $L_{4}$ (Figure 7 ). For the variable camphorated, the multi-set includes only two terms: not and very (Figure 10).

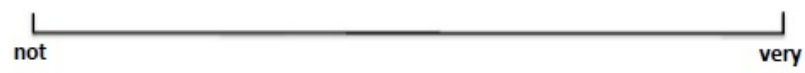

Figure 10. Representation of Camphorated Multi-set

We adapt the expert fuzzy rules to the multi-valued logic context:

- $\mathbf{R}_{\mathbf{1}}$ : If molecule is surely spherical and surface is medium size then odor is very camphorated

- $\mathbf{R}_{\mathbf{2}}$ : If molecule is surely spherical and surface is small size then odor is not camphorated

- $\mathbf{R}_{\mathbf{3}}$ : If molecule is surely spherical and surface is large size then odor is not camphorated

- $\mathbf{R}_{4}$ : If molecule is not spherical then odor is not camphorated
The Table I gives a sample test of 4 molecules from the 99 molecules used.

Table I

A SAMPLE First teSt RESUlts

\begin{tabular}{|c|c|c|c|}
\hline \multicolumn{2}{|c|}{ Inputs } & \multirow{2}{*}{ Odor level } & \multirow{2}{*}{ Result } \\
\cline { 1 - 2 } $\mathbf{R} / \mathbf{r}$ & $\mathbf{S}$ & & \\
\hline 1.90 & 8.96 & very & TP \\
\hline 1.55 & 11.85 & not & FN \\
\hline 2.46 & 11.63 & not & TN \\
\hline 2.17 & 12.97 & not & FN \\
\hline
\end{tabular}

The classification rate of not camphorated molecule is good $(93 \%)$ as all object in this class are homogeneous. However, the very camphorated class includes objects with a weak and strong odor of camphor, i.e. Schnabel score between 1 and 5 . For this reason, we obtain a bad classification rate $(37 \%)$. These results are similar to those obtained in [23] [24].

The accuracy of odor recognition is about $60 \%$ which is not a satisfactory rate.

\section{B. Second Test}

In [23] [24], the authors proposed to consider the sphericity and the density of molecules to deduct the intensity of their camphor odors. The odor is described with two values: not and very. Camphorated perception is done using the following rules:

- if $\mathrm{R} / \mathrm{r}>1.91$ then not camphorated

- if $\mathrm{R} / \mathrm{r} \leq 1.7$ and $\mathrm{d} \leq 0.802$ then very camphorated

In a multi-valued logic context, the inference rules with the multi-sets $L_{3}^{s}$ (Figure 6), $L_{3}^{d}$ (Figure 9 ) and $L_{2}$ (Figure 10) are as follows:

- $\mathbf{R}_{4}$ : If molecule is not spherical then odor is not camphorated

- $\mathbf{R}_{\mathbf{5}}$ : If molecule is surely spherical and molecule is low dense then odor is very camphorated

As in [23] [24], we use in this test only rules $\mathbf{R}_{\mathbf{4}}$ and $\mathbf{R}_{\mathbf{5}}$. A sample of 4 molecules from our test base is presented in Table II.

Table II

A SAMPle SECOND TEST RESUlts

\begin{tabular}{|c|c|c|c|}
\hline \multicolumn{2}{|c|}{ Inputs } & \multirow{2}{*}{ Odor level } & \multirow{2}{*}{ Result } \\
\cline { 1 - 2 } $\mathbf{R} / \mathbf{r}$ & $\mathbf{d}$ & & \\
\hline 1.90 & 0.835 & not & FN \\
\hline 1.55 & 0.795 & very & TP \\
\hline 2.46 & 0.84 & not & TN \\
\hline 2.17 & 0.781 & not & FN \\
\hline
\end{tabular}

The classification rate of non camphorated molecule is insufficient (66\%). However, we obtain a good classification rate $(95 \%)$ for the very camphorated class.

In [23], the accuracy of the non camphorated class is about $93 \%$. While the accuracy of the very camphorated class is about $89 \%$. We notice that we get a lower rate for the first class. While the rate for the second class is slightly better. 
The accuracy of the classification is about $82 \%$ which is better than in the first test.

\section{Third Test}

In this test, we suggest to use the three attributes: surface, sphericity and density not only two of them as in the two first tests. We also propose to distinguish between molecules with a weak and strong camphor odor as in Schnabel et al. [22] work.

We introduce new rules obtained from the set of studied molecules using the new proposed knowledge representation.

The inference rules with the multi-sets $L_{4}$ (Figure 7 ), $L_{3}^{s}$ (Figure 6$), L_{3}^{d}$ (Figure 9 ) and $L_{3}^{c}$ (Figure 8) are:

- $\mathbf{R}_{6}$ : If surface is small size and molecule is low dense then odor is slightly camphorated

- $\mathbf{R}_{\mathbf{7}}$ : If surface is small size and molecule is high dense then odor is not camphorated

- $\mathbf{R}_{\mathbf{8}}$ : If surface is medium size and molecule is low dense then odor is very camphorated

- $\mathbf{R}_{\mathbf{9}}$ : If surface is medium size and molecule is high dense then odor is slightly camphorated

- $\mathbf{R}_{10}$ : If molecule is surely spherical and surface is large size and molecule is low dense then odor is very camphorated

- $\mathbf{R}_{11}$ : If molecule is almost spherical and surface is large size and molecule is low dense then odor is slightly camphorated

- $\mathbf{R}_{12}$ : If molecule is not spherical and surface is large size and molecule is low dense then odor is not camphorated

- $\mathbf{R}_{\mathbf{1 3}}$ : If surface is large size and molecule is high dense then odor is not camphorated

In this test, we use the rules $\mathbf{R}_{\mathbf{6}}$ to $\mathbf{R}_{\mathbf{1 3}}$.

The Table III presents the obtained results for 4 molecules from our test base.

Table III

A SAMPle Third TeSt RESUlts

\begin{tabular}{|c|c|c|c|c|}
\hline \multicolumn{3}{|c|}{ Inputs } & \multirow{2}{*}{ Odor level } & \multirow{2}{*}{ Result } \\
\hline $\mathbf{R} / \mathbf{r}$ & $\mathbf{S}$ & $\mathbf{d}$ & & \\
\hline 1.90 & 8.96 & 0.835 & slightly & TP \\
\hline 1.55 & 11.85 & 0.795 & very & TP \\
\hline 2.46 & 11.63 & 0.84 & not & TN \\
\hline 2.17 & 12.97 & 0.781 & not & FN \\
\hline
\end{tabular}

The obtained classification rates are good for both non camphorated and camphorated molecules, respectively $83 \%$ and $97 \%$. For the camphorated molecule, we obtain a rate of $70 \%$ for slightly camphorated class and $87 \%$ for very camphorated class.

Thus, the accuracy is about $90 \%$ for the classification in two classes non camphorated and camphorated; and $82 \%$ for the classification in three classes non camphorated, slightly camphorated and very camphorated.

\section{Comparative results}

In this paper, we implement a camphor odor classifier within unbalanced multi-sets. We test our tool with three different factual knowledge, i.e. facts representation, and distinct rules set.

We notice that the accuracy of the third test is the best. We also compare these tests using the metrics: precision, recall and F-measure. The table IV illustrates the values of theses metrics for all tests for the classification in two classes: Non camphorated and Camphorated.

The precision, the recall and the F-measure are improved as we use the attributes surface, sphericity and density in the molecule's description and in the new proposed rules. Moreover, the distribution of the camphorated components into two sub-classes, i.e. slightly and very, made the classes more homogeneous. It ameliorates the accuracy of our classification regarding to the first and second tests. The metrics for this test are presented in the Table V.

Table V

Precision Recall F-Measure for the third test

\begin{tabular}{|c|l|l|l|}
\cline { 2 - 4 } \multicolumn{1}{c|}{} & $\begin{array}{l}\text { Not } \\
\text { Camphorated }\end{array}$ & $\begin{array}{l}\text { Slightly } \\
\text { Camphorated }\end{array}$ & $\begin{array}{l}\text { Very } \\
\text { Camphorated }\end{array}$ \\
\hline Cccuracy & \multicolumn{2}{c|}{0.82} \\
\hline Precision & 0.94 & 0.47 & 0.89 \\
\hline Recall & 0.83 & 0.69 & 0.87 \\
\hline F-measure & 0.88 & 0.56 & 0.88 \\
\hline
\end{tabular}

\section{CONCLUSION}

The suitability of unbalanced multi-set theory in camphor odor recognition has been tested in this paper. To do so, an inference engine is implemented to deduct the odor of 99 aliphatic alcohols with one or two carbon atoms. The olfactory properties of molecules used are their measure of sphericity, their measure of surface and their density.

Table IV

Precision Recall F-measure for the three tests

\begin{tabular}{|c|c|c|c|c|c|c|}
\hline & \multicolumn{2}{|c|}{ Test 1} & \multicolumn{2}{|c|}{ Test 2} & \multicolumn{2}{|c|}{ Test 3} \\
\hline & $\begin{array}{l}\text { Not } \\
\text { Camphorated }\end{array}$ & Camphorated & $\begin{array}{l}\text { Not } \\
\text { Camphorated }\end{array}$ & Camphorated & $\begin{array}{l}\text { Not } \\
\text { Camphorated }\end{array}$ & Camphorated \\
\hline Accuracy & 0 & & 0. & & 0 & \\
\hline Precision & 0.49 & 0.875 & 0.9 & 0.8 & 0.94 & 0.89 \\
\hline Recall & 0.925 & 0.35 & 0.66 & 0.95 & 0.83 & 0.96 \\
\hline F-measure & 0.53 & 0.5 & 0.76 & 0.87 & 0.88 & 0.92 \\
\hline
\end{tabular}


Three tests are done using the same training set. Results show that our approach can give similarly (first and second tests), if not better (third test), classification compared to Kissi, Ramdani et al. works.

In the same context of odors recognition, in a future study, we could test our inference engine to detect other odors as sandalwood odor.

In a further work, we will also test the suitability of unbalanced multi-sets in the diagnosis of childhood autism as described in the Childhood Autism Rating Scale (CARS). A tool was already implemented by Bel-Haj Kacem et al. in collaboration with the child psychiatry department of Razzi hospital in Tunisia but it uses uniform multi-sets [25]. An adaptation of this proposal is required to deal with unbalanced multi-sets.

\section{ACKNOWLEDGMENT}

The authors would like to thank Mohamed Kissi and Mohammed Ramdani from the university of Hassan II Mohammedia - Morocco for their help and support in making this work possible by providing us the set of studied compounds.

\section{REFERENCES}

[1] A. G. Bell, Discovery and Invention. National Geographic 649, 1914.

[2] M. Kissi, "Conception et élaboration d'un système expert à base de règles floues pour le traitement d'information chimique," PhD Thesis, Université Paris 6, Université Hassan II Mohammadia, 2004.

[3] T. M. Azahar, S. Shariffah Nur Rasyidah, and C. NurAtiqah Nadiah, "Odor recognition using fuzzy logic algorithm," Journal of Science \&5 Technology JSET, vol. 2, no. Issue 1, 2015.

[4] S. Medasani, J. Kim, and R. Krishnapuram, "An overview of membership function generation techniques for pattern recognition," International Journal of Approximate Reasoning, vol. 19, no. 3, pp. $391-417,1998$.

[5] M. D. Glas, "Knowledge representation in a fuzzy setting," Rapport interne, vol. 89, p. 48, 1989.

[6] H. Akdag and D. Pacholczyk, "Incertitude et logique multivalente, première partie : Etude théorique," BUSEFAL, vol. 38, pp. 122-139, 1989.

[7] H. Akdag, M. D. Glas, and D. Pacholczyk, "A qualitative theory of uncertainty," Fundamenta Informaticae, vol. 17, no. 4, pp. 333-362, 1992

[8] J. Wang and J. Hao, "A new version of 2-tuple fuzzy linguistic representation model for computing with words," IEEE Trans. Fuzzy Systems, vol. 14, no. 3, pp. 435-445, 2006.

[9] E. Herrera-Viedma and A. G. López-Herrera, "A model of an information retrieval system with unbalanced fuzzy linguistic information," Int. J. Intell. Syst., vol. 22, no. 11, pp. 1197-1214, 2007.

[10] F. Herrera, E. Herrera-Viedma, and L. Martínez, "A fuzzy linguistic methodology to deal with unbalanced linguistic term sets," IEEE T. Fuzzy Systems, vol. 16, no. 2, pp. 354-370, 2008.

[11] Y. Dong, Y. Xu, and S. Yu, "Computing the numerical scale of the linguistic term set for the 2-tuple fuzzy linguistic representation model," IEEE Trans. Fuzzy Systems, vol. 17, no. 6, pp. 1366-1378, 2009.

[12] Y. Dong, G. Zhang, W. Hong, and S. Yu, "Linguistic computational model based on 2-tuples and intervals," IEEE Trans. Fuzzy Systems, vol. 21, no. 6, pp. 1006-1018, 2013.

[13] Y. Dong and E. Herrera-Viedma, "Consistency-driven automatic methodology to set interval numerical scales of 2-tuple linguistic term sets and its use in the linguistic GDM with preference relation," IEEE Trans. Cybernetics, vol. 45, no. 4, pp. 780-792, 2015.
[14] I. Truck, "Comparison and links between two 2-tuple linguistic models for decision making," Knowledge-Based Systems, vol. 87, pp. 61-68, Oct. 2015.

[15] M. Xia and Z. Xu, "An approach to multiplicative linguistic group decision making based on possibility degrees," International Transactions in Operational Research, 2015.

[16] L. A. Zadeh, "The concept of a linguistic variable and its application to approximate reasoning," J. Inf. Sci. Vol. 8 (3), and Vol. 8(4) and Vol.9(1), 1975.

[17] F. Khoukhi, "Approche logico-symbolique du traitement des connaissances incertaines et imprécises dans les systèmes à base de connaissances," PhD Thesis, University of Reims Champagne-Ardenne, 1996.

[18] M. El-Sayed and D. Pacholczyk, "Towards a symbolic interpretation of approximate reasoning," Electr. Notes Theor. Comput. Sci., vol. 82, no. 4, pp. 108-119, 2003.

[19] S. B. H. Kacem, A. Borgi, and K. Ghédira, "Generalized modus ponens based on linguistic modifiers in a symbolic multivalued framework," in 38th IEEE International Symposium on Multiple-Valued Logic, May 22-23, 2008, Dallas, Texas, USA, 2008, pp. 150-155.

[20] S. B. H. Kacem, A. Borgi, and M. Tagina, "Extended symbolic approximate reasoning based on linguistic modifiers," Knowl. Inf. Syst., vol. 42, no. 3, pp. 633-661, 2015.

[21] — "Ramoli: A generic knowledge-based systems shell for symbolic data," in 2013 World Congress on Computer and Information Technology (WCCIT), June 2013, pp. 1-6.

[22] K.-O. Schnabel, H.-D. Belitz, and C. von Ranson, "Untersuchungen zur struktur-aktivitäts-beziehung bei geruchsstoffen 1. mitteilung: Wahrnehmungsschwellenwerte und geruchsqualitäten von gesättigten aliphatischen und alicyclischen verbindungen mit sauerstoff-funktion," Zeitschrift für Lebensmittel-Untersuchung und Forschung, vol. 187, no. 3, pp. 215-223, 1988.

[23] M. Kissi, M. Ramdani, M. Tollabi, and D. Zakarya, "Determination of fuzzy logic membership functions using genetic algorithms: application to structure-odor modeling," Journal of Molecular Modeling, vol. 10, no. 5, pp. 335-341, 2004.

[24] M. Ramdani, M. Kissi, and B. Bouchon-Meunier, Intelligent Sensory Evaluation: Methodologies and Applications. Berlin, Heidelberg: Springer Berlin Heidelberg, 2004, ch. Man-Machine Interaction to Extract Features of Odorous Molecules, pp. 255268.

[25] S. B. H. Kacem, A. Borgi, and S. Othman, "A diagnosis aid system of autism in a multi-valued framework," in Uncertainty Modelling in Knowledge Engineering and Decision Making - Proceedings of the 12th International FLINS Conference (FLINS 2016), pp. 405-410. 